\title{
Analgesic effects of Chinese Tuina massage in a rat model of pain
}

\author{
SHICHAO JIANG ${ }^{1-3 *}$, HAO ZHANG $^{1 *}$, MIN FANG $^{1}$, YUQUI ZHANG $^{3}$, NING LU $^{3}$, QINGGUANG ZHU $^{1,2}$, \\ YANBIN CHENG ${ }^{2}$, JIAN AI $^{2}$, NAN ZHOU $^{1}$, JIANHUA LI ${ }^{1}$, LEI FANG ${ }^{4}$ and FEI YAO ${ }^{5}$ \\ ${ }^{1}$ Department of Tuina, Yueyang Hospital of Integrated Traditional Chinese and Western Medicine, \\ Shanghai University of Traditional Chinese Medicine; ${ }^{2}$ Research Institute of Tuina, \\ Shanghai Academy of Traditional Chinese Medicine, Shanghai 200437; Institute of Neurobiology, \\ Institutes of Brain Science and State Key Laboratory of Medical Neurobiology, Fudan University, Shanghai 200032; \\ ${ }^{4}$ School of Rehabilitation Medicine; ${ }^{5}$ College of Acumox and Tuina, Shanghai University of Traditional Chinese Medicine, \\ Shanghai 201210, P.R. China
}

Received October 24, 2014; Accepted October 27, 2015

DOI: 10.3892/etm.2016.3055

\begin{abstract}
Previous clinical trials have suggested that the Chinese Tuina massage may exert transient analgesic effects. However, further investigation regarding the underlying mechanism has been hindered by the lack of a suitable animal model of pain. The present study established a rat model of hind leg pain by injecting 5.8\% hypertonic saline solution (HSS) into the left gastrocnemius muscle. The effects of various Tuina massages on the pain thresholds of the rats were then measured. In addition, the effects of ipsilateral and contralateral Tuina massages on C-fiber-evoked field potentials following electrical stimulation of the left sciatic nerve were determined. Alterations in the gastrocnemius muscle tissues following various Tuina applications were investigated using hematoxylin and eosin, and desmin staining, as well as malondialdehyde and superoxide dismutase assays. Heavy hand pressure transiently reduced the pain sensitivity of both posterior limbs, despite HSS only being injected into the left hind leg. Tuina massage treatments that lasted for 15 min were associated with the best results and an absence of local tissue changes. The results of electrical sciatic nerve stimulation demonstrated that ipsilateral and contralateral Tuina massage may decrease the level of peripheral nociceptive C-fiber activity. In the present study, the Chinese Tuina massage exerted analgesic effects in a rat model of pain, which did not involve tissue damage, following a 15 min massage. Therefore, the rat model of pain used in the
\end{abstract}

Correspondence to: Dr Min Fang, Department of Tuina, Yueyang Hospital of Integrated Traditional Chinese and Western Medicine, Shanghai University of Traditional Chinese Medicine, 110 Ganhe Road, Shanghai 200437, P.R. China

E-mail: fangmin140807@126.com

*Contributed equally

Key words: Tuina massage, C-fiber-evoked field potentials, gastrocnemius muscle, sciatic nerve present study may provide a novel approach for investigating the molecular and physiological mechanisms underlying the therapeutic effects of Tuina massage.

\section{Introduction}

Nociception is a sensory process that leads to pain triggered by nociceptors, which are primary sensory neurons that respond to thermal, mechanical or chemical stimuli (1). There are various major pain theories, including the intensity theory of pain, which postulates that any sensory stimulus with enough intensity can generate pain (2). The peripheral pattern theory suggests that pain is produced by intense stimulation of all skin fiber endings (3), which contradicts the specificity theory, which proposes that there are numerous types of sensory receptors, with each one responding to a specific type of stimuli (4). All of these theories propose that pain is induced by the hyperstimulation of sensory receptors; however, research has suggested that pain may be associated with other sensory stimuli, such as touch (5).

The Chinese Tuina massage is a traditional hand massage, which has previously been associated with pain relief. There are six main styles of physical Tuina therapy, including wobbling, rubbing, vibrating, squeezing, knocking and articular moving. Squeezing involves pressing, pinching, kneading, grasping and rubbing, and is commonly used in Tuina pain treatments (6). Constant softness and penetration under consistent intensities, frequencies and manipulation durations are applied to all styles of Tuina. Clinical practice has demonstrated the therapeutic effects of Tuina for the treatment of pain, depression, chronic inflammation and mechanical injury (7-12). According to the theory of peripheral sensory signaling, there exists an interacting network of sensory nerves in the skin and beneath the muscles (13). Various nerve pathways of the peripheral nervous system participate in distinct signal transductions, and it has previously been demonstrated that acupuncture may attenuate pain signaling by stimulating $A \beta, A \delta$ and C-type nerve fibers (14). Tuina, which selectively stimulates specific nerves, may trigger similar mechanisms, thus explaining its analgesic effects in clinical practice. The present study estab- 
lished a rat model of pain in order to analyze the effects of the Tuina massage on pain threshold.

\section{Materials and methods}

Rats. Male Sprague-Dawley rats (age, 40-50 days; weight, 250-300 g) were purchased from Experimental Animal Center of Fudan University (Shanghai, China) and housed in plastic cages at room temperature, and natural diurnal cycles were applied. All experiments were conducted in compliance with the Institutional Animal Care and Use Committee (Shanghai Municipal Commission of Health and Family Planning), and as few animals were used as possible in order to achieve statistical significance. The present study was approved by the Ethical Committee of the Yueyang Hospital of Integrated Traditional Chinese and Western Medicine (Shanghai, China).

Rat pain models. A total of 20 rats were randomly selected and equally divided into either the $5.8 \%$ hypertonic saline solution (HSS) injection group or the heat-induced pain group. In the HSS group, $0.2 \mathrm{ml} \mathrm{HSS}$ was injected over $30 \mathrm{sec}$ into the center of the left gastrocnemius muscle of the hind limb at a depth of $\sim 0.5 \mathrm{~cm}$. After HSS injection $(0.2 \mathrm{ml})$, the rats in the heat-induced pain group were placed into cages containing pre-heated transparent glass bottoms with Plexiglass panels on all other sides. The rat hind paws were directly heated using a thermal source (Model 336; IITC Life Science Inc., Woodland Hills, CA, USA), the power of which was adjusted according to the weight and size of each individual rat, in order to avoid unnecessary injury. The longest heating period applied was $20 \mathrm{sec}$, and the thermal pain threshold was set between 8 and $12 \mathrm{sec}$ for all rats. Hind paw mechanical withdrawal and paw thermal withdrawal tests were conducted on the bilateral feet, as outlined in previous studies $(15,16)$. Briefly, the rats were housed in a cage containing a metal grid bottom $(20 \times 20 \times 25 \mathrm{~cm})$ and Plexiglass panels. The left paw was teased with a brush pen (Electronic Von Frey; Stoelting Co., Wood Dale, IL, USA), and the paw mechanical withdrawal response was recorded upon contraction of the gastrocnemius muscles. The paw thermal pain withdrawal test was conducted using the Model 33 instrument (IITC Life Science Inc.). Pain thresholds were recorded at 40 and 20 min prior to pain induction (B40 and B20), and 20,40, $60,80,100,120,140$ and $160 \mathrm{~min}$, and at 1 and 2 days (A20, A40, A60, A80, A100, A120, A140, A160, A1D and A2D, respectively) following pain induction.

Tuina procedure. Hand manipulation maneuvers included clockwise pressing and rubbing, with moderate to strong pressure on the skin. Tuina massage was performed on the center of the gastrocnemius muscle for the indicated frequencies and durations. Massage intensity was $80 \%$ of the full potential (at this point, the rats exhibited sensory stimuli feeling without signs of pain and paw withdrawal). Prior to Tuina massage, rats were placed face down on a frame platform to adapt to the experimental environment and to minimize stress. Finger pressure recordings (units in Newton) were monitored using a pressure recording instrument (FingerTPS ${ }^{\mathrm{TM}}$, Pressure Profile Systems, Inc., Tokyo, Japan), as described in a previous study (17).
Experimental design of Tuina massages. Clinically, a Tuina massage lasts for 2-3 min and is repeated 3-5 times with high hand pressure; however, no previous experimental protocol exists for a rat model. In the present study, Tuina massages were performed on the left and right hind gastrocnemius muscles in three groups ( $\mathrm{n}=10$ each), as follows: i) The 5 min group, in which the rats were massaged twice for $2 \mathrm{~min}$ with a $1 \mathrm{~min}$ interval; ii) the 15 min group, in which the rats were massaged for 2 min five times, with 1 min intervals; and iii) the $30 \mathrm{~min}$ group, in which the rats were massaged for 2 min ten times, with 1 min intervals. In the control group, finger skin touch without Tuina was applied. A frequency of $2 \mathrm{~Hz}$ was used for all rats.

Recording of nerve impulses. Rats were anesthetized using $1 \%$ sodium pentobarbital $(40 \mathrm{mg} / \mathrm{kg}$; Beijing Dingguo Changsheng Biotechnology, Co., Ltd., Beijing, China). Following loss of sensation, which was tested by a foot pinch, a cannula was inserted into the trachea of the rats in order to maintain an open airway and low resistance air flow. The spine vertebrae of the rats were clipped for stability and the right hind leg was tied in order to maintain steadiness during the Tuina massage. The body temperature of all rats was maintained between 36.5 and $37.5^{\circ} \mathrm{C}$ using a feedback controlled heating pad, and blood flow in the bilateral hind legs was continuously monitored. A $1.5 \mathrm{~cm}$ skin incision was made along the lower back and over the left femur bone. In order to expose an enlargement of the lumbar spinal cord and sciatic nerve, a T12-L1 laminectomy was performed. A bipolar silver hook electrode was positioned beneath the exposed sciatic nerve and the dorsal spinal cord for physiological and electrophysiological recordings. In addition, the exposed dorsal spinal cord area was filled with a medical wax cotton ball and was stabilized using a warmed paraffin oil pool situated in a skin flap. C-fiber-evoked field potentials were initiated via stimulation of the sciatic nerve using rectangular pulses $(6-18 \mathrm{~V} ; 500 \mu \mathrm{sec}$; interval, $60 \mathrm{sec}$ ), delivered by a silver mercury membrane electrode (Beijing Xinhangxingye, Co., Ltd., Beijing, China). The sciatic nerves were stimulated using $1 \mathrm{~mol} / \mathrm{l} \mathrm{HSS}$ injected through a micropipette, which was fabricated using either a PC-5 N (Narishige Group, Tokyo, Japan) or a laser-based micropipette puller (P-97; Sutter Instrument, Novato, CA, USA), in order to induce a pain response. The T12-L1 nerve impulse signals were displayed on an oscilloscope (VC-11; Nihon Kohden, Tokyo, Japan), which was connected to a Pentium computer (Intel, Santa Clara, CA, USA) via a CED 1401 interface for off-line analysis using Spike2 software (Cambridge Electronic Design Ltd., Cambridge, UK). Data are presented as C-fiber evoked field potentials and nerve impulse thresholds at a defined baseline (\%).

Immunohistochemical staining for tissue damage. In order to assess the extent of tissue damage following Tuina massage, treated rats were sacrificed via cervical dislocation at the end of the experiments, and the gastrocnemius muscle tissues of 12 rats from the 5,15 and 30 min Tuina groups were removed ( $\mathrm{n}=4$ each). Sections of gastrocnemius muscle tissue $(0.5 \times 0.5 \mathrm{~cm})$ were mounted on glass slides, fixed with acetone for $10 \mathrm{~min}$ and subsequently washed three times with $0.01 \mathrm{M}$ phosphate-buffered saline (PBS), after which the tissue 
sections were blocked with 5\% sheep serum (Experimental Animal Center of Fudan University) at room temperature for 20 min. Mouse anti-desmin monoclonal primary antibody (dilution, 1:5; cat. no. BM0036; Wuhan Boster Biological Technology, Ltd., Wuhan, China) was added to the slides and incubated at $37^{\circ} \mathrm{C}$ for $1 \mathrm{~h}$, after which the slides were washed three times with 0.01 M PBS (pH 7.2-7.6). The secondary fluorescein isothiocyanate-conjugated anti-mouse immunoglobulin G antibody (dilution, 1:100; cat. no. SA1062; Wuhan Boster Biological Technology Co., Ltd.), was added to the slides, which were subsequently incubated in a dark room for 2-3 $\mathrm{h}$ at room temperature. The tissue sections were washed with PBS, and 4',6-diamidino-2-phenylindole was added for $10 \mathrm{~min}$ in order to stain cell nuclei. After washing three times with PBS, sections were mounted in buffered glycerin and were visualized under a fluorescence microscope (Nikon Eclipse E600; Nikon Corp., Tokyo, Japan) within 24 h.

Hematoxylin and eosin $(H \& E)$ staining. For $H \& E$ staining of muscle sections, slices were incubated twice with xylene, for 10 min each time, followed by rehydration twice in absolute alcohol (5 min each time) and then twice in 95 and $75 \%$ alcohol (2 min each time). Next, the slices were stained with Harris-modified hematoxylin solution (Bogu Biological Science and Technology Co., Ltd., Shanghai, China) for $8 \mathrm{~min}$, then rinsed with distilled water for $10 \mathrm{~min}$, after which $1 \%$ hydrochloric acid-alcohol solution was added for $30 \mathrm{sec}$ followed by washing with distilled water for $1 \mathrm{~min}$. Subsequently, $0.2 \%$ ammonia water saturated lithium carbonate (Yuanmu Biological Science and Technology Co. Ltd., Shanghai, China) was added for 30-60 sec, and the slides were washed again with distilled water for $5 \mathrm{~min}$. Finally, the samples were counterstained with eosin-phloxine solution (Bogu Biological Science and Technology Co., Ltd.) for 30-60 sec subsequent to washing with 10 drops of $95 \%$ alcohol. Following dehydration with absolute alcohol and $95 \%$ alcohol twice for 5 min each time, the samples were cleared twice in xylene ( 5 min each time) and mounted with xylene-based mounting medium (Yuanmu Biological Science and Technology Co. Ltd., Shanghai, China).

Malondialdehyde (MDA) and superoxide dismutase (SOD) assays. In order to measure the concentrations of MDA and SOD in the gastrocnemius muscle tissue sections, $0.1 \mathrm{~g}$ muscle samples were cut into small pieces. Subsequently, the tissues were homogenized in $0.9 \mathrm{ml}$ radioimmunoprecipitation assay cell lysis buffer (Shanghai Changdao Biotechnology, Co., Ltd., Shanghai, China) with an ultrasonic homogenizer (Qsonica, LLC, Newtown, CT, USA) and were maintained on ice for $20 \mathrm{~min}$. Following centrifugation at $900 \mathrm{x} \mathrm{g}$ for $10 \mathrm{~min}$, the supernatant was removed for further experimentation.

MDA protein expression levels were measured using the thiobarbituric acid reaction method. Briefly, samples were heated at $95^{\circ} \mathrm{C}$ in a water bath for $40 \mathrm{~min}$ and were subsequently cooled with tap water, after which $200 \mu$ l samples were added to a 96-well plate, followed by substrate reagents (Sigma-Aldrich, Beijing, China) at $37^{\circ} \mathrm{C}$, to allow color development. Optical density (OD) was measured using an Epoch spectrometer (Biotek Instruments, Inc., Winooski, VT, USA) at a wavelength of $532 \mathrm{~nm}$. The MDA concentration was calculated using the following equation: $\mathrm{MDA}(\mathrm{nmol} / \mathrm{mg}$ protein $)=$
[(Sample $\mathrm{OD}_{532}$-Control $\left.\mathrm{OD}_{532}\right) /\left(\right.$ Standard $\mathrm{OD}_{532}$-Control $\left.\mathrm{OD}_{532}\right)$ ] x standard concentration $(10 \mathrm{nmol} / \mathrm{ml}) / \mathrm{sample}$ protein concentration (mg protein $/ \mathrm{ml}$ ).

SOD protein expression levels were measured using the WST-1 method, according to the manufacturer's protocol (Dojindo Laboratories, Kumamoto, Japan). Briefly, $200 \mu 1$ protein samples were added into a 96-well plate, after which substrate reagent was added at $37^{\circ} \mathrm{C}$ for $20 \mathrm{~min}$. OD was measured using a Epoch spectrometer (Biotek Instruments, Inc.) at $450 \mathrm{~nm}$. The SOD concentration was calculated using the following equation: SOD inhibitory rate $=100 \times\left[\left(\right.\right.$ control- control $\left.^{\Delta \mathrm{C}}\right)-\left(\right.$ sample- sample $\left.\left.^{\Delta \mathrm{T}}\right)\right] /$ [control-control ${ }^{\Delta \mathrm{C}}$ ], where $\mathrm{C}$ is the difference between the control and the control blank, and $\mathrm{T}$ is the difference between the sample and the sample blank. The SOD activity was calculated using the following equation: SOD activity $(\mathrm{U} / \mathrm{mg}$ protein $)=\mathrm{SOD}$ inhibiting rate $/ 50 \% \mathrm{x}$ dilution/protein concentration.

Statistical analysis. Data analyses were performed using Sigmastat 3.5 softwate (Systat Software, Inc., San Jose, CA, USA). Mean mechanical and thermal pain threshold values pre-Tuina were calculated using the B40 and B20 groups, respectively. Post-Tuina pain threshold percentages were calculated using the following equation: Post-Tuina $(\%)=$ (post-Tuina mean value/pre-Tuina mean value) $x 100 \%$. One-way repeated analysis of variance (ANOVA), followed by a Student-Newman-Keuls test, was conducted in order to compare the pain withdrawal threshold values between the pre- and post-Tuina groups. In addition, the pain withdrawal threshold values between each time point were compared using two-way repeated ANOVA, followed by Student-Newman-Keuls test. Data are presented as the mean pain withdrawal threshold value \pm standard error. The areas under the curves (AUC) of the C-fiber evoked field potentials are presented as the mean \pm standard error of four time points, and were analyzed using two-way repeated ANOVA, followed by Student-Newman-Keuls test. The SOD and MDA concentrations in the gastrocnemius muscle are presented as the mean \pm standard error of four groups. Data were analyzed using one-way ANOVA, and Tamhane's T2 polynomial test was used for multiple comparisons. $\mathrm{P}<0.05$ was considered to indicate a statistically significant difference.

\section{Results}

HSS (5.8\%), but not thermal pain, reduces the mechanical pain threshold. In order to establish a rat model of pain, $5.8 \%$ HSS was injected into the left gastrocnemius muscle of the rats (Fig. 1). Decreased mechanical pain threshold values and hypersensitivity were detected in the injected and contralateral gastrocnemius muscles of the A20, A40, A60, A80, A100, A120, A140, A160, A1D and A2D group rats $(n=10)$; thus suggesting that $5.8 \%$ HSS was able to decrease the mechanical pain threshold value and induce pain for 2 days post-injection (Fig. 1). Conversely, the thermal pain threshold of the bilateral gastrocnemius muscles was not altered until $160 \mathrm{~min}$ following 5.8\% HSS injection; however, significantly elevated thermal pain thresholds were detected at $1-2$ days post-injection in the left muscle ( $\mathrm{P}<0.05$; Fig. 2$)$. 
Table I. Mechanical pain threshold in the ipsilateral leg following Tuina massage (mean\% $\pm \mathrm{SE}$ ).

\begin{tabular}{lcccc}
\hline Group & Non-Tuina & Tuina 5 min & Tuina 15 min & 10 \\
\hline $\mathrm{N}$ & 10 & 10 & 10 & $1.03 \pm 0.13$ \\
$\mathrm{~B} 40$ & $0.98 \pm 0.06$ & $1.06 \pm 0.14$ & $1.02 \pm 0.14$ & $0.97 \pm 0.13$ \\
$\mathrm{~B} 20$ & $1.02 \pm 0.06$ & $0.94 \pm 0.14$ & $0.98 \pm 0.14$ & $1.16 \pm 0.82^{\mathrm{b}}$ \\
$\mathrm{A} 20$ & $0.98 \pm 0.21$ & $1.14 \pm 0.25$ & $1.32 \pm 0.38^{\mathrm{a}}$ & $0.88 \pm 0.22$ \\
$\mathrm{~A} 40$ & $1.00 \pm 0.19$ & $1.15 \pm 0.30$ & $1.36 \pm 0.41^{\mathrm{c}}$ & $0.92 \pm 0.21$ \\
$\mathrm{~A} 60$ & $0.96 \pm 0.22$ & $1.14 \pm 0.31$ & $1.23 \pm 0.30$ & $0.83 \pm 0.38$ \\
$\mathrm{~A} 80$ & $0.94 \pm 0.23$ & $1.14 \pm 0.32$ & $1.25 \pm 0.29$ & $0.68 \pm 0.22$ \\
$\mathrm{~A} 1 \mathrm{D}$ & $1.00 \pm 0.20$ & $0.98 \pm 0.27$ & $1.03 \pm 0.39^{\mathrm{d}}$ & $0.71 \pm 0.17$ \\
$\mathrm{~A} 2 \mathrm{D}$ & $1.00 \pm 0.30$ & $0.97 \pm 0.13$ & $1.01 \pm 0.31^{\mathrm{e}}$ & \\
\hline
\end{tabular}

Tuina 15 min group: ${ }^{\mathrm{a} B} 20$ vs. $\mathrm{A} 20, \mathrm{P}=0.042$; ${ }^{\mathrm{B}} \mathrm{B} 20$ vs. $\mathrm{A} 40, \mathrm{P}=0.012$; ${ }^{\mathrm{A}} \mathrm{A} 40$ vs. $\mathrm{A} 1 \mathrm{D}, \mathrm{P}=0.025$; ${ }^{\mathrm{e}} \mathrm{A} 40$ vs. A2D, $\mathrm{P}=0.016$; Tuina 30 min group: ${ }^{\mathrm{b}} \mathrm{B} 20$ vs. A20, $\mathrm{P}=0.025$. B, before HSS injection; A, after HSS injection; HSS, hypertonic saline solution; SE, standard error.

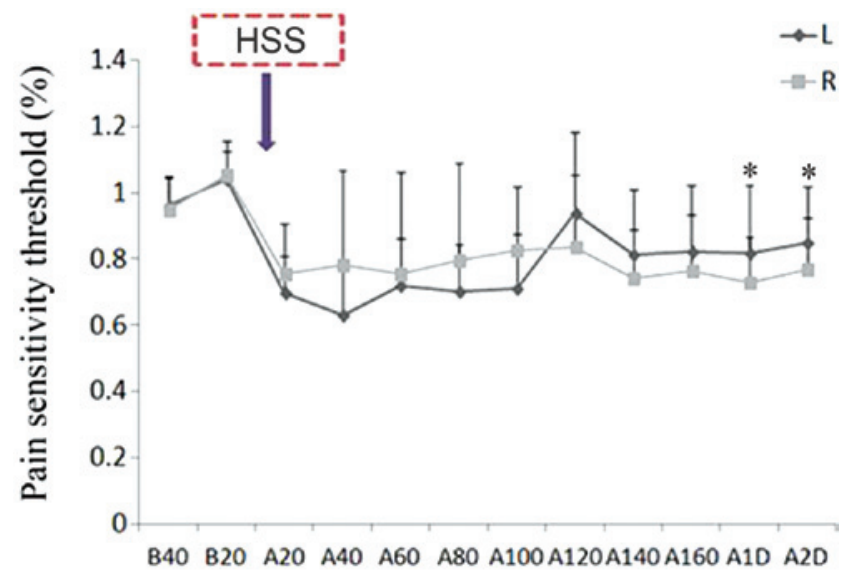

Figure 1. HSS (5.8\%) injection decreased the mechanical pain threshold value of the bilateral hind legs of the rats, and did not recover until 2 days post-injection $(n=10)$. Left leg: B20 vs. A20-A100, ${ }^{*} \mathrm{P}<0.01$; $\mathrm{B} 20$ vs. A140-A2D, ${ }^{*} \mathrm{P}<0.05$. Right leg: B20 vs. A20-A80 and A14-A2D, ${ }^{*} \mathrm{P}<0.01$; $\mathrm{B} 20$ vs. A100 and A120, "P<0.05. L, left leg; R, right leg; B, before HSS injection; A, after HSS injection; HSS, hypertonic saline solution.

Therefore, $5.8 \%$ HSS treatment, but not thermal heat, may be considered a useful strategy for developing a rat model of pain.

Tuinamassagefor 15 min increases mechanicalpain thresholds in the right gastrocnemius muscle following injection of $5.8 \%$ HSS into the left gastrocnemius muscle. In order to investigate whether Tuina affected the mechanical pain sensitivity and threshold values of contralateral muscles following HSS injection, a 15 min Tuina massage was performed on the right gastrocnemius muscles of the treated rats. Tuina treatment for 15 min significantly increased the mechanical pain thresholds in the right muscle at 20 and 40 min following Tuina massage, as compared with the non-massaged control group rats; however, the elevated thresholds reached a plateau at 1 day post-Tuina massage. In the 30 min Tuina massage group with high hand pressure, the mechanical pain threshold of the right muscle increased within 20 min of treatment; however, it had decreased at 1-2 days post-Tuina massage, as compared with the A20 group (Table I and Fig. 3).

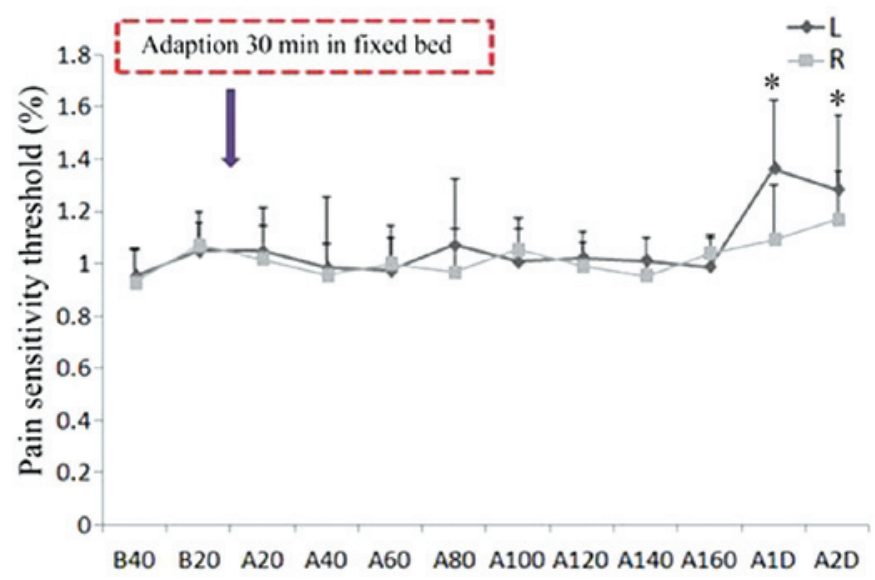

Figure 2. Thermal pain threshold values were increased 1 day following thermal stimulation $(\mathrm{n}=10)$. Left leg: ${ }^{*} \mathrm{P}<0.01$, A1D vs. B40-A160; ${ }^{*} \mathrm{P}<0.05$, A2D vs. B40-A160. Right leg: *P<0.05, A2D vs. B40-A140. L, left leg; $\mathrm{R}$, right leg; $\mathrm{B}$, before pain induction; $\mathrm{A}$, after pain induction.

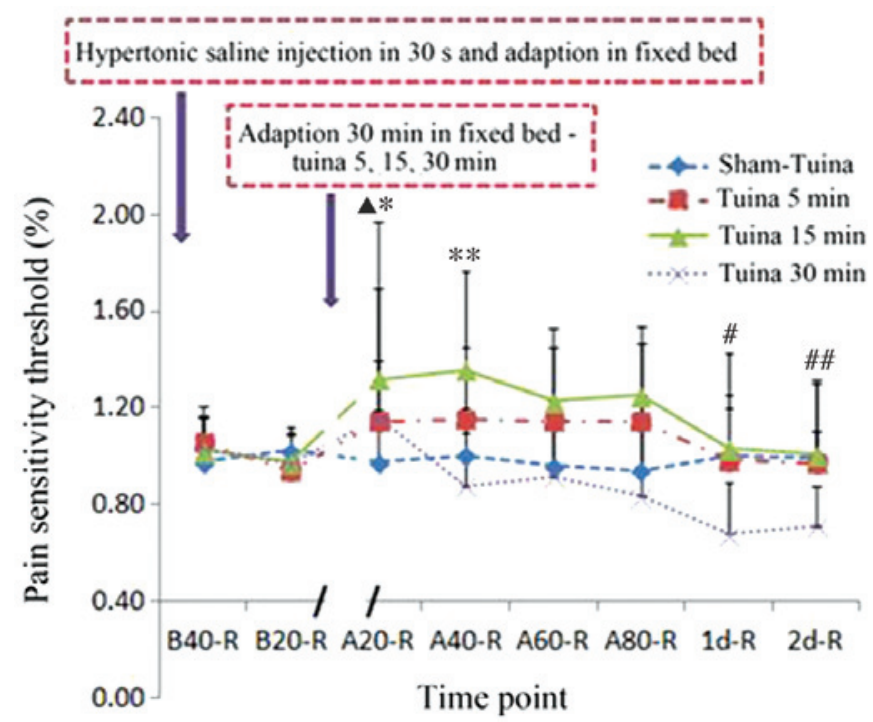

Figure 3. Tuina massage affects the mechanical pain threshold of the ipsilateral leg. Tuina 15 min group: ${ }^{*} \mathrm{P}<0.05$, B20 vs. A20; ${ }^{* *} \mathrm{P}<0.05, \mathrm{~B} 20$ vs. A40; ${ }^{\#} \mathrm{P}<0.05, \mathrm{~A} 40$ vs. $1 \mathrm{~d} ;{ }^{\# \#} \mathrm{P}<0.05$, A40 vs. 2 d. Tuina 30 min group: ${ }^{\star} \mathrm{P}<0.05, \mathrm{~B} 20$ vs. A20. B, before-Tuina; A, after-Tuina; R, right leg. 
Table II. Mechanical pain threshold in the contralateral leg following Tuina massage (mean $\% \pm \mathrm{SE}$ ).

\begin{tabular}{lcccc}
\hline Group & Non-Tuina & Tuina 5 min & Tuina 15 min & 10 \\
\hline $\mathrm{N}$ & 10 & 10 & 10 & $0.99 \pm 0.12$ \\
$\mathrm{~B} 40$ & $0.95 \pm 0.13$ & $1.03 \pm 0.18$ & $1.06 \pm 0.15$ & $1.01 \pm 0.12$ \\
$\mathrm{~B} 20$ & $1.05 \pm 0.13$ & $0.97 \pm 0.18$ & $0.94 \pm 0.15$ & $1.33 \pm 0.30^{\mathrm{b}, \mathrm{c}}$ \\
$\mathrm{A} 20$ & $0.99 \pm 0.14$ & $0.92 \pm 0.37$ & $1.40 \pm 0.37^{\mathrm{a}}$ & $1.20 \pm 0.24$ \\
$\mathrm{~A} 40$ & $0.83 \pm 0.21$ & $0.98 \pm 0.42$ & $1.36 \pm 0.40^{\mathrm{d}}$ & $1.08 \pm 0.29$ \\
$\mathrm{~A} 60$ & $1.01 \pm 0.13$ & $1.01 \pm 0.42$ & $1.23 \pm 0.38^{\mathrm{e}}$ & $1.05 \pm 0.14$ \\
$\mathrm{~A} 80$ & $0.87 \pm 0.19$ & $0.96 \pm 0.37$ & $0.98 \pm 0.22$ & $0.79 \pm 0.16$ \\
A1D & $0.96 \pm 0.16$ & $0.95 \pm 0.17$ & $0.98 \pm 0.15$ & $0.89 \pm 0.25$ \\
A2D & $0.96 \pm 0.12$ & $0.94 \pm 0.19$ & &
\end{tabular}

Tuina 15 min group: ${ }^{\mathrm{B}} \mathrm{B} 20$ vs. $\mathrm{A} 20, \mathrm{P}<0.001$; ${ }^{\mathrm{B}} \mathrm{B} 20$ vs. $\mathrm{A} 40, \mathrm{P}<0.001$; ${ }^{\mathrm{e}} \mathrm{B} 20$ vs. $\mathrm{A} 60, \mathrm{P}=0.02 ;{ }^{\mathrm{f}} \mathrm{B} 20$ vs. A80, $\mathrm{P}=0.022$. Tuina 30 min group: ${ }^{\mathrm{b}} \mathrm{B} 20$ vs. A20, $\mathrm{P}=0.003$; ${ }^{\mathrm{B}} \mathrm{B} 40$ vs. A20, $\mathrm{P}=0.003$. B, before HSS injection; A, after HSS injection; HSS, hypertonic saline solution; SE, standard error.

Tuina massage of the right gastrocnemius muscle for $15 \mathrm{~min}$ increases the mechanical pain threshold of the left gastrocnemius muscle in HSS-injected rats. In order to investigate whether Tuina massage of the right gastrocnemius muscle was able to affect the pain sensitivity and threshold of the left contralateral muscle, 15 min Tuina was performed on the right gastrocnemius muscles of HSS-treated rats and the pain sensitivity of the left gastrocnemius muscles were measured. An increased pain threshold was detected for the left muscle at $80 \mathrm{~min}$ post-Tuina, and was shown to plateau at 1 day post-Tuina, as compared with the non-Tuina control groups. Similarly, 30 min Tuina massage was associated with an increased pain threshold at $20 \mathrm{~min}$ post-Tuina (Table II and Fig. 4).

Muscle damage was not detected following Tuina massage for 5 and $15 \mathrm{~min}$; however, Tuina massage performed for $30 \mathrm{~min}$ was shown to induce moderate damage to the treated muscles. In order to investigate whether Tuina massage caused damage to the gastrocnemius muscles, the muscles were removed immediately following Tuina massage treatments for histological and immunohistochemical analyses. There were no detectable histological alterations in the Tuina 15 and 20 min groups, as compared with the non-Tuina controls (Figs. 5A-C and 6A-C). However, in the 30 min Tuina group, abnormal muscle fibers, minor swelling and necrotic areas were observed.

Tuina increases SOD content, but not MDA content, in the bilateral gastrocnemius muscles. In order to investigate whether damaged muscle tissues post-Tuina were associated with altered SOD and MDA protein expression levels, SOD and MDA concentrations were measured in the bilateral gastrocnemius muscles. Tuina massage for 15 and $30 \mathrm{~min}$ significantly increased the levels of SOD, but not MDA, in the bilateral gastrocnemius muscles, as compared with the ipsilateral muscle tissue in the non-Tuina group; thus suggesting that Tuina massage may induce moderate biochemical alterations (Table III).

Tuina inhibits harmful spinal dorsal horn C-fiber activity. C-fiber evoked field potentials in the superficial spinal dorsal horn were recorded, and the results of the Tuina $15 \mathrm{~min}$ and non-Tuina control groups were compared. Local 5.8\% HSS injection into the left gastrocnemius muscle was found to

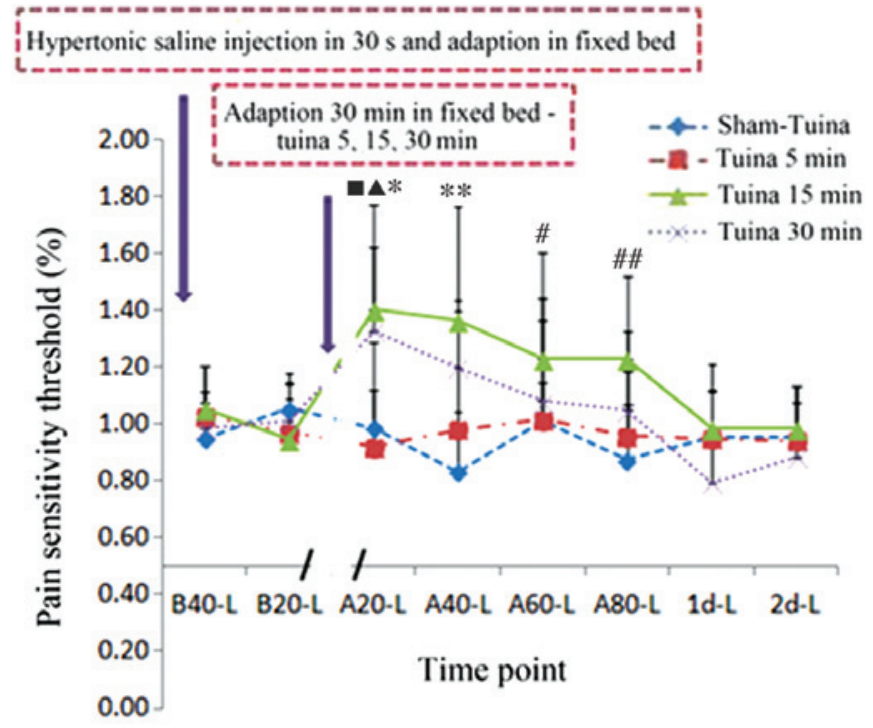

Figure 4. Tuina massage affects the mechanical pain threshold of the contralateral leg. Hypertonic saline solution (5.8\%) injection decreased the left leg pain threshold, which was reversed following adaptation in a fixed bed and Tuina massage using high hand pressure. Tuina 15 min group: ${ }^{*} \mathrm{P}<0.001$, B20 vs. A20; ${ }^{* *} \mathrm{P}<0.001, \mathrm{~B} 20$ vs. A40; ${ }^{\#} \mathrm{P}<0.05$, B20 vs. A60; ${ }^{\# \#} \mathrm{P}<0.05, \mathrm{~B} 20$ vs. A80. Tuina 30 min group: ${ }^{\mathbf{\Delta}} \mathrm{P}<0.05$, B20 vs. A20; ${ }^{-\mathrm{P}<0.05}$, B40 vs. A20. $\mathrm{B}$, before-Tuina; A, after-Tuina; L, left leg.

be associated with a decreased peak amplitude and AUC of the sciatic nerves. However, subsequent to Tuina massage for $15 \mathrm{~min}$, the peak amplitude of the C-fiber discharge and the AUC of the ipsilateral and contralateral muscles were significantly decreased, when compared with those in the control group $(\mathrm{P}<0.001$; Table IV and Fig. 7). The aforementioned results suggested that Tuina is able to decrease the sensitivity of the $\mathrm{C}$-fibers in the sciatic nerves of the bilateral gastrocnemius muscles. Similar results were detected for the other Tuina groups, including the A20, A40, A60 and A80 groups.

\section{Discussion}

Tuina massage, which has been used in China for clinical purposes for $>1,000$ years, is an effective therapeutic physical 
Table III. SOD and MDA content in bilateral gastrocnemius muscles.

\begin{tabular}{lcccc}
\hline Group & Non-Tuina & Tuina 5 min & Tuina 15 min & Tuina 30 min \\
\hline N & 8 & & & 8 \\
Left & 8 & 8 & 7 & 8 \\
Right & & 8 & & \\
SOD (U/mg prot) & $0.68 \pm 0.08$ & $1.03 \pm 0.19$ & $2.13 \pm 0.22^{\mathrm{a}}$ & $1.81 \pm 0.43^{\mathrm{b}}$ \\
Left & $0.59 \pm 0.11$ & $0.79 \pm 0.13$ & $1.48 \pm 0.22$ & $2.11 \pm 0.53^{\mathrm{c}}$ \\
Right & & & & \\
MDA (nmol/mg prot) & $0.64 \pm 0.08$ & $0.57 \pm 0.07$ & $1.18 \pm 0.32$ & $0.83 \pm 0.13$ \\
Left & $0.79 \pm 0.06$ & $0.61 \pm 0.07$ & $0.68 \pm 0.05$ & $0.70 \pm 0.04$ \\
Right &
\end{tabular}

${ }^{\mathrm{a}} \mathrm{P}=0.004$, vs. the ipsilateral hind leg of the non-Tuina group; ${ }^{\mathrm{b}} \mathrm{P}=0.014$, vs. the ipsilateral hind leg of the non-Tuina group; ${ }^{\mathrm{C}} \mathrm{P}=0.007$, vs. the ipsilateral hind leg of the non-Tuina group. SOD, superoxide dismutase; MDA, malondialdehyde.
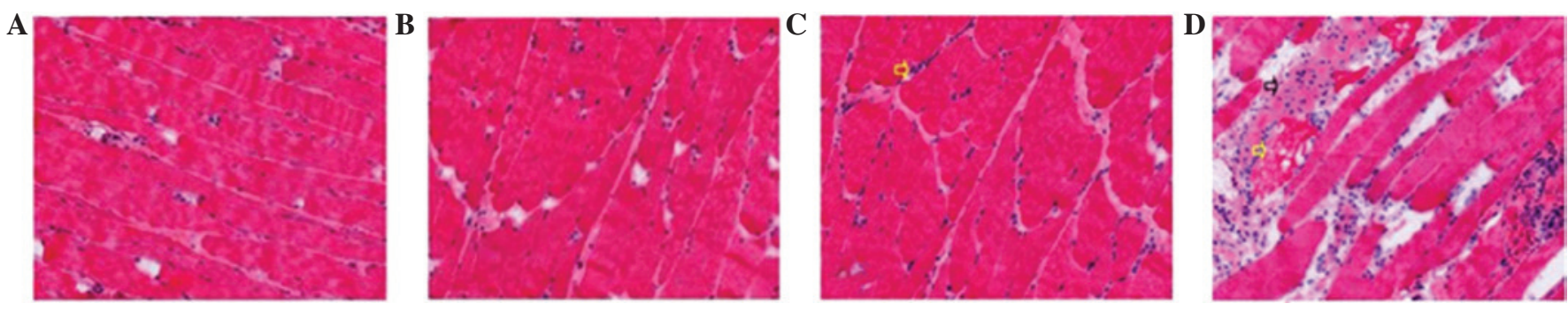

Figure 5. Muscle tissue staining with hematoxylin and eosin. (A) Non-Tuina group and (B) 15 min Tuina group exhibited no marked alterations in tissue morphology: Long and cylindrical multi-nuclear skeletal muscle cells were in a compact arrangement; the stripes with light and dark areas were clear, regular and repetitive; the nucleus under the cell membrane was flat, oval and of normal size; the nucleus appeared distributed around the sarcoplasm; and no cell swelling, atrophy or inflammatory cell infiltration were detected. The (C) 20 min Tuina group was similar in appearance to the non-Tuina and 15 min Tuina groups; however, a small amount of nuclear cell aggregation was detected (yellow arrow). In the (D) 30 min Tuina group, cytoplasm staining was significantly decreased and cytolysis (black arrow), local nucleus aggregation (yellow arrow) and infiltration of inflammatory cells were detected, suggesting mild edema of the muscle fiber.
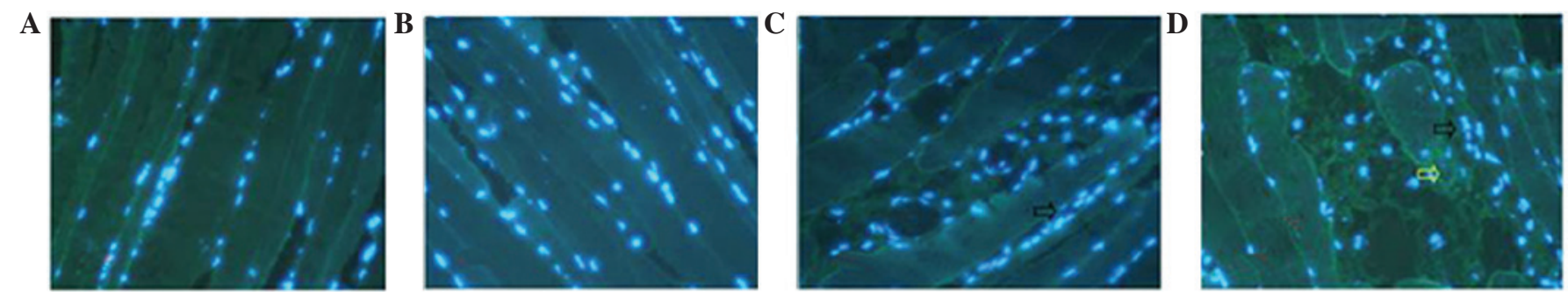

Figure 6. Muscle histology following staining with anti-desmin at various Tuina time points. (A and B) Non-Tuina massage group; (C) 15 min Tuina massage group; and (D) 30 min Tuina massage group. Yellow arrow, skeletal muscle cell cytoskeleton; black arrow, nucleosis.

massage that provides pain relief and helps to alleviate the symptoms of chronic inflammatory diseases (18). However, the underlying mechanism remains obscure, due to the lack of experimental animal models. The present study established a rat model of pain via intramuscular injection of $5.8 \% \mathrm{HSS}$, in order to reduce mechanical pain thresholds. This effect was reversed by $15 \mathrm{~min}$ Tuina massage treatment of the ipsilateral and contralateral gastrocnemius muscles. The results of the present study suggested that the spinal dorsal horn C-fiber responses of the sciatic nerve were reduced following Tuina massage, as demonstrated by a modified protocol for recording C-fiber evoked field potentials $(15,16)$. The underlying physiological and biochemical mechanisms underlying Tuina massage-induced analgesic effects are currently unknown. However, a cross-talk between the skin and the underlying muscles is provided by peripheral sensory systems. Impulses are transmitted from skin sensors to the central nervous system via the spinal cord and various nerve impulses are transmitted by distinct nerve sensors and fibers. According to the gate-control theory, small nerve fibers transmit pain signals and large nerve fibers transmit normal signals to the brain (19). However, both types of nerve fiber interact with projection cells, which extend through the spinothalamic tract to the brain and to inhibitory interneurons within the 

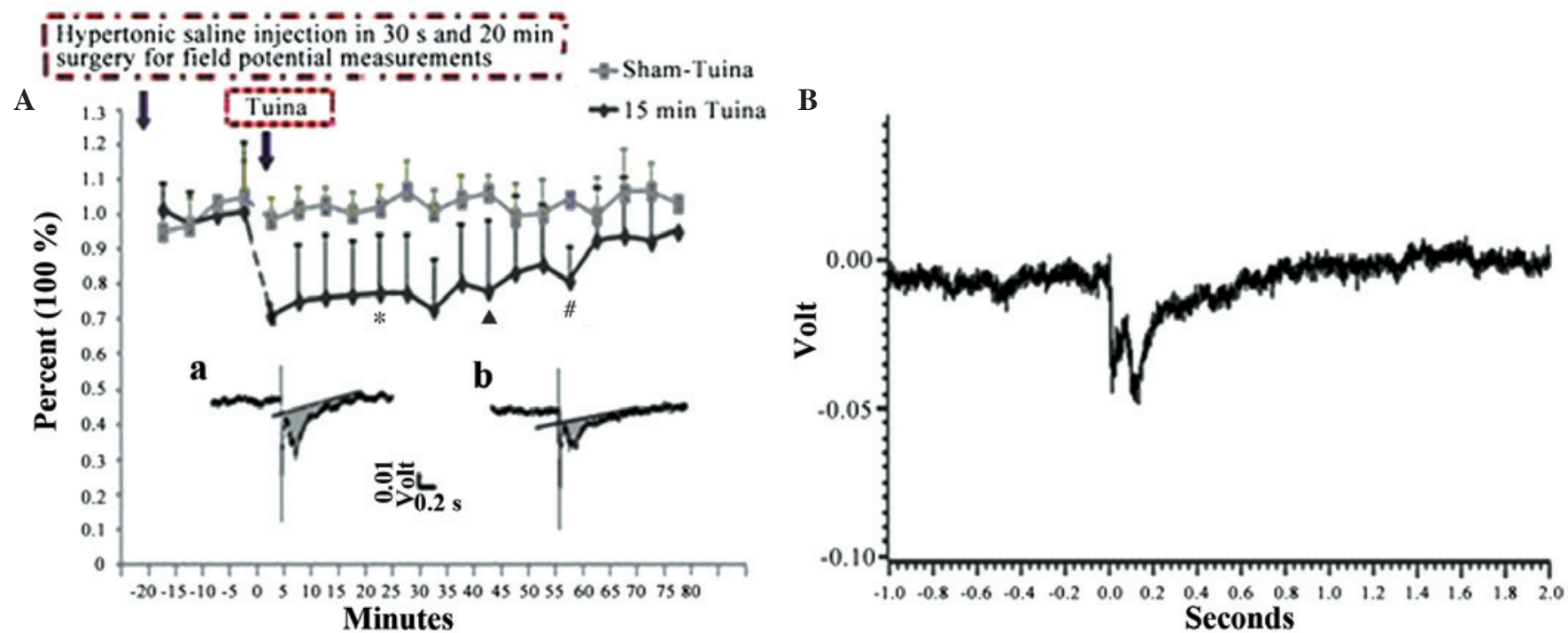

Figure 7. Hypertonic saline solution was injected into the gastrocnemius muscles of the left rat hind legs. (A) Alterations in the area under the tangent of the spinal cord dorsal horn field C-fiber evoked field potentials were recorded following Tuina intervention. Representative original recordings (mean of six consecutive traces) taken (a) 18 min prior to injection and (b) $1 \mathrm{~h}$ following tetanic stimulation. The gray area under the tangent was calculated and used for statistical analyses. (B) Representative image of a single pulse derived from the spinal cord dorsal horn field potential. At 20 min, "P<0.05 vs. Tuina group; at $40 \mathrm{~min},{ }^{\wedge} \mathrm{P}<0.05$ vs. non-Tuina control group; at $60 \mathrm{~min},{ }^{,} \mathrm{P}<0.05$ vs. non-Tuina control group.

Table IV. Spinal dorsal horn C-fiber-evoked field potential in sciatic nerves following $5.8 \%$ HSS injection (mean\% \pm standard error).

\begin{tabular}{lcc}
\hline Group & Non-Tuina & Tuina $15 \mathrm{~min}$ \\
\hline $\mathrm{N}$ & 3 & 8 \\
$\mathrm{~B} 20$ & $0.95 \pm 0.06$ & $1.02 \pm 0.10$ \\
$\mathrm{~A} 20$ & $1.00 \pm 0.05$ & $0.77 \pm 0.18^{\mathrm{a}}$ \\
$\mathrm{A} 40$ & $1.04 \pm 0.06$ & $0.80 \pm 0.15^{\mathrm{b}}$ \\
$\mathrm{A} 60$ & $1.05 \pm 0.10$ & $0.81 \pm 0.18^{\mathrm{c}}$ \\
$\mathrm{A} 80$ & $1.03 \pm 0.08$ & $0.95 \pm 0.17$ \\
\hline
\end{tabular}

${ }^{\mathrm{a}} \mathrm{P}<0.008$, vs. non-Tuina group; ${ }^{\mathrm{b}} \mathrm{P}<0.017$, vs. non-Tuina group; ${ }^{\mathrm{c}} \mathrm{P}<0.026$, vs. non-Tuina group. B, before HSS injection; A, after HSS injection; HSS, hypertonic saline solution.

dorsal horn (19). Pain signals from the projection neurons are transmitted to the brain upon the inactivation of inhibitory neurons, which is thought to occur when small-fiber stimulation is dominant. In the case of predominant long nerve fiber signaling, activated inhibitory neurons prevent the projection neurons from sending signals to the brain (19).

The present study hypothesized that Tuina massage may induce long nerve fiber signaling and thereby inhibit pain signaling to the central nervous system by the activation of inhibitory neurons. Low frequency electroacupuncture has previously been shown to reduce C-fiber-evoked nerve volleys in the dorsal horn following sciatic nerve stimulation. However, this effect was inhibited by the opioid receptor antagonist naloxone; thus suggesting that endogenous opioids may also have a role in modifying pain signals (20). Future studies should investigate the underlying mechanisms of Tuina massage modified pain signaling.
In conclusion, the present study demonstrated an analgesic effect for middle point gastrocnemius muscle Tuina massage in a rat model of pain. The optimal application mode was $2 \mathrm{~Hz}$ for $15 \mathrm{~min}$, consisting of 2 min cycles and 1 min intervals between cycles. No alterations were detected in the gastrocnemius muscle morphology following a 15 min Tuina massage. The pain relief effects of Tuina were associated with elevated pain thresholds and reduced AUC of C-fiber-evoked field potentials of the ipsilateral and contralateral nerves.

\section{Acknowledgements}

The present study was supported by grants from the National Science Foundation for Distinguished Young Scholars (grant nos. 81025022 and 81503673), the budgetary funds of the Project of Shanghai University of Traditional Chinese Medicine (grant no. 2013JW30), the 'Sailing program' of the Shanghai Science and Technology Committee (grant no. 14YF1411700), Research Projects in the Industry of Traditional Chinese Medicine (grant no. 2015468003-1) and Research Projects from Shanghai Municipal Commission of Health and Family Planning (grant no. 20154Y0175).

\section{References}

1. Dubin AE and Patapoutian A. Nociceptors: The sensors of the pain pathway. J Clin Invest 120: 3760-3772, 2010.

2. Norrsell U, Finger S and Lajonchere C: Cutaneous sensory spots and the 'law of specific nerve energies': History and development of ideas. Brain Res Bull 48: 457-465, 1999.

3. Sinclair DC. Cutaneous sensation and the doctrine of specific energy. Brain 78: 584-614, 1955.

4. Moayedi M and Davis KD. Theories of pain: From specificity to gate control. J Neurophysiol 109: 5-12, 2013.

5. Melzack R and Wall PD. Pain mechanisms: A new theory. Science 150: 971-979, 1965.

6. YU TY: Science of Chinese massage. Chinese Medicine Press: 88-95, 2013. 
7. Cherkin DC, Sherman KJ, Kahn J, Wellman R, Cook AJ, Johnson E, Erro J, Delaney K and Deyo RA: A comparison of the effects of 2 types of massage and usual care on chronic low back pain: A randomized, controlled trial. Ann Intern Med 155: 1-9, 2011.

8. Astin JA and Ernst E: The effectiveness of spinal manipulation for the treatment of headache disorders: A systematic review of randomized clinical trials. Cephalalgia 22: 617-623, 2002.

9. Degirmen N, Ozerdogan N, Sayiner D, Kosgeroglu N and Ayranci U: Effectiveness of foot and hand massage in postcesarean pain control in a group of Turkish pregnant women. Appl Nurs Res 23: 153-158, 2010.

10. Mitchinson AR, Kim HM, Rosenberg JM, Geisser M, Kirsh M, Cikrit D and Hinshaw DB: Acute postoperative pain management using massage as an adjuvant therapy: A randomized trial. Arch Surg 142: 1158-1167; discussion 1167, 2007.

11. Piotrowski MM, Paterson C, Mitchinson A, Kim HM, Kirsh M and Hinshaw DB: Massage as adjuvant therapy in the management of acute postoperative pain: A preliminary study in men. J Am Coll Surg 197: 1037-1046, 2003.

12. Sherman KJ, Cherkin DC, Hawkes RJ, Miglioretti DL and Deyo RA: Randomized trial of therapeutic massage for chronic neck pain. Clin J Pain 25: 233-238, 2009.
13. Katz B: Depolarization of sensory terminals and the initiation of impulses in the muscle spindle. J Physiol 111: 261-282, 1950.

14. Zhao ZQ: Neural mechanism underlying acupuncture analgesia. Prog Neurobiol 85: 355-375, 2008.

15. Schouenborg J and Weng HR: Sensorimotor transformation in a spinal motor system. Exp Brain Res 100: 170-174, 1994.

16. Ferreira SH, Lorenzetti BB and Corrêa FM: Central and peripheral antialgesic action of aspirin-like drugs. Eur J Pharmacol 53: 39-48, 1978.

17. Randall LO and Selitto JJ: A method for measurement of analgesic activity on inflamed tissue. Arch Int Pharmacodyn Ther 111: 409-419, 1957.

18. Ilic D, Djurovic A, Brdareski Z, Vukomanovic A, Pejovic V and Grajic M: The position of chinese massage (Tuina) in clinical medicine. Vojnosanit Pregl 69: 999-1004, 2012.

19. Melzack R and Wall PD: Pain mechanisms: A new theory. Science 150: 971-979, 1965

20. Xing GG, Liu FY, Qu XX, Han JS and Wan Y: Long-term synaptic plasticity in the spinal dorsal horn and its modulation by electroacupuncture in rats with neuropathic pain. Exp Neurol 208: 323-332, 2007. 